\title{
Structures of S-Matrices for Three Body Schrödinger Operators
}

\author{
Hiroshi Isozaki \\ Department of Mathematics, Osaka University, Toyonaka, 560, Japan
}

Received September 9, 1991

\begin{abstract}
Structures of the S-matrix associated with the collision process from 2 clusters to 3 clusters are studied. This S-matrix is shown to have a continuous kernel except for 2-dimensional spheres on which 2-body subsystems have zero velocity. On these spheres, the S-matrix has, in general, singularities whose existence arises from the zero eigenvalues and the zero resonances of the 2-body subsystems.
\end{abstract}

\section{Introduction}

1.1 Collision Process in the Three-Body Problem. We consider collision processes of quantum mechanical three particles labelled by $1,2,3$. Suppose in the initial state the two of them form a bound state, denoted by $(1,2)$, and the third particle collides with this pair. Then it follows from the asymptotic completeness of the wave operators (see e.g. Enss [4], Sigal-Soffer [20] or Graf [7]) that there occurs one of the following five phenomena:

$$
(1,2)+(3) \Rightarrow \begin{cases}\text { (a) } & (1,2)+(3) \\ \text { (b) } & (1,2)^{*}+(3)_{*} \\ \text { (c) } & (1,2)^{\prime}+(3) \\ \text { (d) } & (1,3)+(2) \\ \text { (e) } & (1)+(2)+(3) .\end{cases}
$$

(a) is an elastic process. In (b), the energy of the pair changes. In (c), the energy of the pair does not change, but this pair takes a different state (which happens when the eigenvalue is degenerate). (d) is a rearrangement process. Finally in (e), all of the three particles move freely after the collision. The first four cases are treated in essentially the same way as in the 2-body problem. In this paper, we study properties of the S-matrix associated with the case (e).

$1.2 S$-matrix. In $\mathbf{R}^{3}$ we consider three particles with mass $m_{i}$ and position $x^{i}$. We 
choose a pair $(i, j)$ and denote it by $\alpha$. Let

$$
\frac{1}{m_{\alpha}}=\frac{1}{m_{i}}+\frac{1}{m_{j}}, \quad \frac{1}{n_{\alpha}}=\frac{1}{m_{k}}+\frac{1}{m_{i}+m_{j}}
$$

be the reduced masses and

$$
x^{\alpha}=\sqrt{2 m_{\alpha}}\left(x^{i}-x^{j}\right), \quad x_{\alpha}=\sqrt{2 n_{\alpha}}\left(x^{k}-\frac{m_{i} x^{i}+m_{j} x^{j}}{m_{i}+m_{j}}\right)
$$

be the relative coordinates. Let

$$
X=\left\{\left(x^{1}, x^{2}, x^{3}\right) ; \sum_{i=1}^{3} m_{i} x^{i}=0\right\}
$$

Then in $L^{2}(X)$ the Schrödinger operator is given by

$$
H=H_{0}+\sum_{\alpha} V_{\alpha}\left(x^{\alpha}\right), \quad H_{0}=-\Delta_{x^{x}}-\Delta_{x_{x}}
$$

If the pair potentials decay faster than $\left|x^{\alpha}\right|^{-1}$ as $\left|x^{\alpha}\right| \rightarrow \infty$, the wave operators are known to exist:

$$
\begin{aligned}
& W_{0}^{ \pm}=\underset{t \rightarrow \pm \infty}{s-\lim e^{i t H} e^{-i t H_{0}}} \\
& W_{\alpha}^{ \pm}=\underset{t \rightarrow \pm \infty}{s-\lim _{t \rightarrow \infty} e^{i t H} e^{-i t H_{x}} J_{\alpha}}
\end{aligned}
$$

where

$$
H_{\alpha}=H_{0}+V_{\alpha}, \quad\left(J_{\alpha} f\right)\left(x^{\alpha}, x_{\alpha}\right)=u_{\alpha}\left(x^{\alpha}\right) f\left(x_{\alpha}\right),
$$

$u_{\alpha}$ being a normalized eigenfunction of $h^{\alpha}=-\Delta_{x^{x}}+V_{\alpha}\left(x^{\alpha}\right)$ with eigenvalue $E^{\alpha}<0$. The scattering operator $S_{0 \alpha}$ is defined by

$$
S_{0 \alpha}=\left(W_{0}^{+}\right) * W_{\alpha}^{-} .
$$

To introduce the S-matrix, we use unitary operators

$$
\mathscr{F}_{0}: L^{2}\left(\mathbf{R}^{6}\right) \rightarrow L^{2}\left((0, \infty) ; L^{2}\left(S^{5}\right)\right)
$$

and

$$
\mathscr{F}_{\alpha}: L^{2}\left(\mathbf{R}^{3}\right) \rightarrow L^{2}\left(\left(E^{\alpha}, \infty\right) ; L^{2}\left(S^{2}\right)\right)
$$

defined by

$$
\begin{aligned}
\left(\mathscr{F}_{0} f\right)(\lambda, \theta) & =C_{0}(\lambda) \int_{\mathbf{R}^{6}} e^{-i \sqrt{\lambda} \theta \cdot x} f(x) d x, \\
C_{0}(\lambda) & =(2 \pi)^{-3} 2^{-1 / 2} \lambda, \\
\left(\mathscr{F}_{\alpha} f\right)(\lambda, \omega) & =C_{\alpha}(\lambda) \int_{\mathbf{R}^{3}} e^{-i \sqrt{\lambda-E^{\alpha}} \omega \cdot x} f(x) d x, \\
C_{\alpha}(\lambda) & =(2 \pi)^{-3 / 2} 2^{-1 / 2}\left(\lambda-E^{\alpha}\right)^{1 / 4} .
\end{aligned}
$$

Let

$$
\hat{S}_{0 \alpha}=\mathscr{F}_{0} S_{0 \alpha} \mathscr{F}_{\alpha}^{*} .
$$


Then as is well-known (see e.g. Reed-Simon [18]), $\hat{S}_{0 \alpha}$ is decomposable, namely, for any $\lambda>0$, there exists a bounded operator $\hat{S}_{0 \alpha}(\lambda) \in \mathbf{B}\left(L^{2}\left(S^{2}\right) ; L^{2}\left(S^{5}\right)\right)$ such that

$$
\left(\hat{S}_{0 \alpha} f\right)(\lambda, \theta)=\left(\hat{S}_{0 \alpha}(\lambda) f(\lambda, \cdot)\right)(\theta)
$$

for a.e. $\lambda>0, \theta \in S^{5}$ and all $f \in L^{2}\left(\left(E^{\alpha}, \infty\right) ; L^{2}\left(S^{2}\right)\right)$. This $\hat{S}_{0 \alpha}(\lambda)$ is called the S-matrix. Note that this definition contains a sort of ambiguity. Two families of operators $\left\{\hat{S}_{0 \alpha}(\lambda)_{i}\right\}_{\lambda=0}^{\infty}(i=1,2)$ define the same scattering operator $S_{0 \alpha}$, if $\hat{S}_{0 \alpha}(\lambda)_{1}=\hat{S}_{0 \alpha}(\lambda)_{2}$ for a.e. $\lambda>0$. The study of this family of operators $\left\{\hat{S}_{\alpha}(\lambda)\right\}_{\lambda=0}^{\infty}$ is not an easy problem. The general result known so far is that of Amrein-Pearson-Sinha [1] and EnssSimon [5] asserting that $\hat{S}_{0 \alpha}(\lambda)$ is a Hilbert-Schmidt operator for a.e. $\lambda>0$, if the pair potentials decay faster than $\left|x^{\alpha}\right|^{-2}$. In this paper, we shall show that there is a representative $\left\{\hat{S}_{0 \alpha}(\lambda)\right\}_{\lambda=0}^{\infty}$ continuous in $\lambda>0$ and investigate its detailed properties.

1.3. Main Results. We assume that $V_{\alpha}$ is a real $C^{\infty}$-function such that for a constant $\rho>0$,

$$
\left|\partial_{y}^{m} V_{\alpha}(y)\right| \leqq C_{m}(1+|y|)^{-\rho-m}, \quad m=0,1,2, \ldots,
$$

where $\partial_{y}^{m}$ denotes an arbitrary derivative of $m^{\text {th }}$ order with respect to $y$, and $C_{m}$ is a constant. This assumption is stronger than actually needed. One can also allow certain local singularities for $V_{\alpha}$. Let

$$
X_{\beta}=\left\{x \in X ; x^{\beta}=0\right\}
$$

and define

$$
M=S^{5} \backslash \cup_{\beta} X_{\beta}, \quad N=S^{5} \cap\left(\cup_{\beta} X_{\beta}\right) .
$$

Theorem 1.1. (1) Suppose $\rho>4+1 / 2$. Then $\hat{S}_{0 \alpha}(\lambda)$ has a continuous kernel outside $N$ :

$$
\hat{S}_{0 \alpha}(\lambda ; \theta, \omega) \in C\left((0, \infty) \times M \times S^{2}\right) .
$$

(2) Suppose $\rho>5+1 / 2$. Let $\beta$ be any pair and decompose $\theta \in S^{5}$ as $\theta=\left(\theta^{\beta}, \theta_{\beta}\right)$ in accordance with the choice of the Jacobi-coordinates. Then as $\left|\theta^{\beta}\right| \rightarrow 0$,

$$
\hat{S}_{0 \alpha}(\lambda ; \theta, \omega) \simeq\left|\theta^{\beta}\right|^{-1} A_{\beta,-1}\left(\lambda ; \frac{\theta^{\beta}}{\left|\theta^{\beta}\right|}, \theta_{\beta}, \omega\right)+A_{\beta, 0}\left(\lambda ; \frac{\theta^{\beta}}{\left|\theta^{\beta}\right|}, \theta_{\beta}, \omega\right),
$$

where

$$
\begin{aligned}
A_{\beta,-1}\left(\lambda ; \frac{\theta^{\beta}}{\left|\theta^{\beta}\right|}, \theta_{\beta}, \omega\right)= & \sum_{j}^{\text {finite }} C_{\beta 1}^{(j)}\left(\lambda ; \theta_{\beta}, \omega\right) \times \int_{\mathbf{R}^{3}} \frac{\theta^{\beta}}{\left|\theta^{\beta}\right|} \cdot x^{\beta} V_{\beta}\left(x^{\beta}\right) u_{\beta}^{(j)}\left(x^{\beta}\right) d x^{\beta} \\
& +C_{\beta 2}\left(\lambda ; \theta_{\beta}, \omega\right) \times \int_{\mathbf{R}^{3}} V_{\beta}\left(x^{\beta}\right) \varphi_{\beta}\left(x^{\beta}\right) d x^{\beta}
\end{aligned}
$$

$u_{\beta}^{(j)}$ being the eigenfunction with zero eigenvalue for $h^{\beta}$, and $\varphi_{\beta}$ the zero-resonance. $A_{\beta, 0}$ is continuous with respect to all of its arguments. $A_{\beta,-1}=0$, if 0 is neither an eigenvalue nor the resonance for $h^{\beta}$. In this case, $\hat{S}_{0 \alpha}(\lambda ; \theta, \omega)$ is continuous at $\theta^{\beta}=0$.

In the course of the proof, we shall see that $\hat{S}_{0 \alpha}(\lambda)$ is a $\mathbf{B}\left(L^{2}\left(S^{2}\right) ; L^{2}\left(S^{5}\right)\right)$-valued continuous function of $\lambda>0$ if $\rho>5+1 / 2$. The zero-resonance $\varphi_{\beta}$ is the solution of the equation $h^{\beta} \varphi_{\beta}=0$ which behaves like $\varphi_{\beta} \cong C /\left|x^{\beta}\right|$ as $\left|x^{\beta}\right| \rightarrow \infty, C \neq 0$. From our proof given below, one can see that if the pair potentials decay sufficiently rapidly, $\hat{S}_{0 \alpha}(\lambda ; \theta, \omega)$ is smooth on $M \times S^{2}$, but the zero eigenvalue and the zero- 
resonance are known to exist even if the potential is compactly supported. (See e.g. [3].) For the S-matrix from 2 to 3 cluster scattering, it is therefore the zeroeigenfunctions and the zero-resonances of subsystems that determines its singularities.

As for the coefficients $C_{\beta 1}^{(j)}\left(\lambda ; \theta_{\beta}, \omega\right)$ and $C_{\beta 2}\left(\lambda ; \theta_{\beta}, \omega\right)$, we have

Theorem 1.2. Up to a multiplicative constant depending only on $\lambda$ and $E^{\alpha}, C_{\beta 1}^{(j)}\left(\lambda ; \theta_{\beta}, \omega\right)$ and $C_{\beta 2}\left(\lambda ; \theta_{\beta}, \omega\right)$ coincide with the scattering amplitudes for two cluster scattering.

More precisely, $C_{\beta 1}^{(j)}\left(\lambda ; \theta_{\beta}, \omega\right)$ and $C_{\beta 2}\left(\lambda ; \theta_{\beta}, \omega\right)$ are the scattering amplitudes for 2-cluster scattering in which, after the collision, the pair $\beta$ becomes the bound state with zero energy or the zero-resonance, respectively. One should note that the notion of 2-cluster scattering associated with the zero-resonance is somewhat ambiguous since, as far as the author knows, this notion has not yet been introduced in mathematical literature. We shall explain the situation in Sect. 5.

1.4. Methods. To prove Theorem 1.1 we use the method employed by [11] whose key idea is to localize the S-matrix in the phase space. To control the resolvent of $H$, we utilize the estimate of Skibsted [21] established recently on propagation properties in the phase space of $e^{-i t H}$. The singularities of $\hat{S}_{0 \alpha}(\lambda)$ arise from the lowenergy asymptotics of 2-body subsystems studied by Jensen and Kato [13]. To prove Theorem 1.2, in particular the assertion for the zero-resonance, we study spatially asymptotic properties of generalized eigenfunctions of $H$. This point of view is continued in our forthcoming paper [10] to derive all scattering amplitudes with initial state of 2-clusters.

1.5. Remarks. Amrein, Pearson and Sinha [1] showed that, for the N-body problem, the total cross-section with 2-cluster initial state is finite for almost all energy and derived its asymptotic properties in an averaged sense under the assumption that the potentials decay faster than $\left|x^{\alpha}\right|^{-2}$. See also Enss-Simon [5]. Amrein and Sinha [2] also showed that, for the three body problem, the total cross-section is finite for all $\lambda>0$ under the assumption that each 2-body sybsystem has neither the zero eigenvalue nor the zero-resonance. Ito and Tamura [12] studied the semi-classical asymptotics for the total cross-section in distributional sense. All of these works treats the case of the initial state of 2-clusters, while Yafaev [22] studied the structure of the S-matrix, in a two Hilbert space setting, which contains the collision process from 3-clusters to 3-clusters.

1.6. Plan of the Paper. In Sect. 2, we prepare the basic estimates for the resolvent of $H$ and also the low-energy asymptotic expansion of the 2-body problem. Section 3 is devoted to deriving a localization formula of the S-matrix. Theorems 1.1 and 1.2 are proved in Sects. 4 and 5, respectively.

The notation used in this paper is almost standard. For $x \in \mathbf{R}^{n}$ we put $\langle x\rangle=$ $\left(1+|x|^{2}\right)^{1 / 2}$. For Banach spaces $X_{1}$ and $X_{2}, \mathbf{B}\left(X_{1}, X_{2}\right)$ denotes the totality of bounded operators from $X_{1}$ to $X_{2}$. C... is used to denote various constants. $L^{2, s}$ denotes the space of measurable functions such that

$$
\|f\|_{s}^{2}=\int_{\mathbf{R}^{n}}\langle x\rangle^{2 s}|f(x)|^{2} d x<\infty
$$




\section{Preliminaries}

2.1. Functional Calculus. We start with a simple functional calculus. In Subsects. 2-1 and 2-2, we assume that $\rho>0, \rho$ being defined in the assumption (1.9). We consider a pseudo-differential operator (Ps.D.Op.) $P$ with symbol $p(x, \xi)$ having the following properties:

$$
\left|\partial_{x}^{m} \partial_{\xi}^{n} p(x, \xi)\right| \leqq C_{m n}\langle x\rangle^{-m}, \text { for any } m, n \geqq 0 .
$$

There exists a closed cone $\Gamma \subset X \backslash \cup_{\alpha} X_{\alpha}$ such that

$$
\operatorname{supp}_{x} p(x, \xi) \subset \Gamma \text { for all } \xi \text {. }
$$

Lemma 2.1. Let $P$ be a Ps.D.Op. with symbol $p(x, \xi)$ satisfying (2.1) and (2.2). Let $\varphi(\lambda) \in C_{0}^{\infty}\left(\mathbf{R}^{1}\right)$. Then for any $N \geqq 1$, there exist $\varphi_{1}, \ldots, \varphi_{N} \in C_{0}^{\infty}\left(\mathbf{R}^{1}\right)$ and $p_{1}(x, \xi), \ldots$, $p_{N}(x, \xi)$ satisfying $(2.1)$ and (2.2) such that $\operatorname{supp} \varphi_{m} \subset \operatorname{supp} \varphi(m=1, \ldots, N)$, and

$$
\begin{aligned}
p\left(x, D_{x}\right) \varphi(H) & =p\left(x, D_{x}\right) \varphi\left(H_{0}\right)+\sum_{m=1}^{N}\langle x\rangle^{-\rho m} p_{m}\left(x, D_{x}\right) \varphi_{m}\left(H_{0}\right)+R_{n}, \\
& \langle x\rangle^{\rho N / 2} R_{N}\langle x\rangle^{\rho N / 2} \in \mathbf{B}\left(L^{2}(X) ; L^{2}(X)\right) .
\end{aligned}
$$

This lemma is proved in the same way as in Theorem 2.1 of [9]. From the very proof, one can see that $\langle x\rangle^{-\rho m} p_{m}(x, \xi)$ consists of a polynomial of derivatives of $p(x, \xi),|\xi|^{2}$ and $V_{\alpha}$ and also that $\varphi_{m}$ consists of derivatives of $\varphi$.

2.2. Resolvent Estimates. Let $R(z)=(H-z)^{-1}$ and $\Lambda$ be the set of thresholds of $H$, which is known to be a countable closed set and $\Lambda \cap(0, \infty)=\varnothing([6])$. Then by the well-known result of Mourre [15] and Perry-Simon [17], we have

$$
\langle x\rangle^{-s} R(\lambda \pm i 0)\langle x\rangle^{-s} \in \mathbf{B}\left(L^{2}(X) ; L^{2}(X)\right),
$$

if $s>1 / 2, \lambda \in \sigma_{e}(H)-\Lambda, \sigma_{e}(H)$ denoting the essential spectrum of $H$.

Another important estimate needed in this paper is that of Skibsted. We consider a Ps.D.Op. $P_{-}$with symbol $p_{-}(x, \xi)$ satisfying $(2.1)$ and $(2.2)$ and also the following:

There exists a constant $\mu_{-}$such that $-1<\mu_{-}<1$ and

$$
p_{-}(x, \xi)=0 \text { if } \hat{x} \cdot \hat{\xi}>\mu_{-},
$$

where $\hat{x}=x /|x|, \hat{\xi}=\xi /|\xi|$. Then we have

Theorem 2.2. Let $P_{-}$be a Ps.D.Op. with symbol $p_{-}(x, \xi)$ satisfying (2.1), (2.2) and (2.4). Then

$$
\langle x\rangle^{s} P_{-} R(\lambda+i 0)\langle x\rangle^{-s-t} \in \mathbf{B}\left(L^{2}(X) ; L^{2}(X)\right)
$$

if $s>-1 / 2, t>1, \lambda \in \sigma_{e}(H)-\Lambda$.

This theorem is essentially due to Skibsted [21]. However, we should add some explanations. Let $I$ be a compact interval contained in $\sigma_{e}(H)-\Lambda$, and $\varphi \in C_{0}^{\infty}(I)$. Then what Skibsted showed is that for $0<s\left\langle s^{\prime}\right.$, the operator $P_{-} \varphi(H) e^{-i t H}\langle x\rangle^{-s^{\prime}}$ has the decay rate $(1+t)^{-s}$ when $t \rightarrow \infty([21]$, Theorem 4.4). By repeating his arguments one can see that for $s \geqq 0,0<\mu^{\prime}<\mu$, the operator $\langle x\rangle^{s} P_{-} \varphi(H) e^{-i t H}\langle x\rangle^{-s-\mu}$ has the decay rate $(1+t)^{-\mu^{\prime}}$ when $t \rightarrow \infty$. Passing to the Laplace transform, we have

$$
\langle x\rangle^{s} P_{-} \varphi(H) R(\lambda+i 0)\langle x\rangle^{-s-\mu} \in \mathbf{B}\left(L^{2}(X) ; L^{2}(X)\right)
$$


for $s \geqq 0, \mu>1$. We then interpolate (2.3) and (2.5) to obtain

$$
\langle x\rangle^{s} P_{-} \varphi(H) R(\lambda+i 0)\langle x\rangle^{-s-t} \in \mathbf{B}\left(L^{2}(X) ; L^{2}(X)\right)
$$

for $s>-1 / 2, t>1$. The theorem then follows from Lemma 2.1 and (2.6). One can also see in (2.3) and Theorem 2.2 the operator norms are uniformly bounded in $\lambda$, if $\lambda$ varies over a compact set in $\sigma_{e}(H)-\Lambda$.

2.3. Zero-Resonances. We review the result of Jensen-Kato on the low-energy asymptotic expansion of 2-body Schrödinger operators. Let $H_{2}=-\Delta+V(x)$ in $\mathbf{R}^{3}$. Suppose that $V(x)$ is a bounded real function and

$$
V(x)=O\left(|x|^{-\rho}\right), \quad|x| \rightarrow \infty, \quad \rho>2 .
$$

By the zero-resonance we mean a solution $\varphi$ of the equation $-\Delta \varphi+V \varphi=0$ in $\mathbf{R}^{3}$ which behaves like $\varphi \cong C /|x|, C \neq 0,|x| \rightarrow \infty$. Let $P_{0}$ be the projection onto the eigenspace of $H_{2}$ with zero eigenvalue. Let $B_{-2}, B_{-1}$ be defined by

$$
\begin{gathered}
B_{-2}=-P_{0}, \\
B_{-1}=-i P_{0} V G V P_{0}+i\langle\cdot, \varphi\rangle \varphi,
\end{gathered}
$$

where $G$ is an integral operator with kernel $|x-y|^{2} /(24 \pi)$. Then by [13], if $\rho>5$ and $s>5 / 2$, we have the following asymptotic expansion of $R_{2}(z)=\left(H_{2}-z\right)^{-1}$.

$$
R_{2}(z)=\frac{B_{-2}}{z}+\frac{B_{-1}}{\sqrt{z}}+O(1), \quad \text { as } \quad z \rightarrow 0,
$$

in $\mathbf{B}\left(L^{2, s} ; L^{2,-s}\right) . P_{0}$ is known to be finite dimensional. If $B_{-2}=B_{-1}=0$. We have a better result:

$$
R_{2}(z)=B_{0}+\sqrt{z} B_{1}+o(\sqrt{z}), \quad \text { as } \quad z \rightarrow 0,
$$

in $\mathbf{B}\left(L^{2, s} ; L^{2,-s}\right), s>3 / 2, \rho>3$. The expansions (2.10) and (2.11) depend largely on the space dimension, and one can also obtain the complete asymptotic expansion. See [16].

We end this section by studying some properties of eigenfunctions of $\mathrm{H}_{2}$ with zero eigenvalue.

Lemma 2.3. Suppose $\rho>3+1 / 2$. Let $u$ be an eigenfunction of $\mathrm{H}_{2}$ with zero eigenvalue. Then

(1) $\int_{\mathbf{R}^{3}} e^{-i x \cdot \xi} V(x) u(x) d x=-i \int_{\mathbf{R}^{3}} x \cdot \xi V(x) u(x) d x+O\left(|\xi|^{2}\right)$, as $|\xi| \rightarrow 0$.

(2) $u(x)=O\left(|x|^{-2}\right)$ as $|x| \rightarrow \infty$.

Proof. (1) We have only to show

$$
\int_{\mathbf{R}^{3}} V(x) u(x) d x=0 .
$$

Let $v=V u=\Delta u$. By passing to the Fourier transform, $\hat{v}(\xi)=-|\xi|^{2} \hat{u}(\xi)$. Since $v \in L^{1}\left(\mathbf{R}^{3}\right), \hat{v}(\xi)$ is continuous. This shows that as $|\xi| \rightarrow 0, \hat{u}(\xi) \cong-\hat{v}(0)|\xi|^{-2}$. Since $u \in L^{2}\left(\mathbf{R}^{3}\right), \hat{v}(0)$ must vanish, which proves $(2.12)$. 
(2) $u$ satisfies the integral equation

$$
u(x)=-\frac{1}{4 \pi} \int_{\mathbf{R}^{3}} \frac{1}{|x-y|} V(y) u(y) d y .
$$

We put $\omega(x, y)=\frac{1}{|x-y|}-\frac{1}{|x|}$. Then using (2.12), we have

$$
u(x)=\frac{1}{4 \pi} \int_{\mathbf{R}^{3}} w(x, y) V(y) u(y) d y .
$$

We put $r=|x|, \hat{x}=x / r$. If $|y|<r / 2$, we have

$$
|w(x, y)|=r^{-1}|| \hat{x}-\left.\frac{y}{r}\right|^{-1}-1|\leqq C| y \mid r^{-2} \text {. }
$$

Therefore,

$$
\int_{|y|<r / 2}|w(x, y) V(y) u(y)| d y \leqq C r^{-2} \int|y| \cdot|V(y) u(y)| d y \leqq C r^{-2} .
$$

The integral over the region $\{y ;|y| \geqq r / 2\}$ is split into two parts:

$$
\int_{|y|>r / 2}|w(x, y) V(y) u(y)| d y \leqq \int_{|y|>r / 2}|x-y|^{-1}|V(y) u(y)| d y+r^{-1} \int_{|y|>r / 2}|V(y) u(y)| d y .
$$

Using the decay assumption on the potential,

$$
r^{-1} \int_{|y|>r / 2}|V(y) u(y)| d y \leqq C r^{-2} \int_{|y|>r / 2}(1+|y|)^{1-\rho}|u(y)| d y \leqq C r^{-2},
$$

and

$$
\int_{|y|>2 r}|x-y|^{-1}|V(y) u(y)| d y \leqq C r^{-1} \int_{|y|>2 r}|V(y)||u(y)| d y \leqq C r^{-2} .
$$

Finally

$$
\begin{aligned}
\int_{r / 2<|y|<2 r}|x-y|^{-1}|V(y) u(y)| d y & \leqq C r^{-\rho} \int_{r / 2<|y|<2 r}|x-y|^{-1}|u(y)| d y \\
& \leqq C r^{-\rho}\left(\int_{r / 2<|y|<2 r}|x-y|^{-2} d y\right)^{1 / 2} \\
& \leqq C r^{-\rho+1 / 2},
\end{aligned}
$$

which completes the proof.

\section{Localization of $\hat{S}_{0 \alpha}$}

In this section, we derive a formula of localization of $\hat{S}_{0 \alpha}$ in the phase space. To localize the direction of propagation of particles, we take a real-valued function $\psi(\theta) \in C^{\infty}\left(S^{5}\right)$. To localize the energy, we take a compact interval $I \subset(0, \infty)$ and a real-valued function $\psi_{0}(t) \in C_{0}^{\infty}((0, \infty))$ such that $\psi_{0}(t)=1$ on $I$. Let $\chi(x, \xi)$ be a 
smooth function satisfying (2.1) and

$$
\chi(x, \xi)=1,
$$

if $|\xi|^{2} \in \operatorname{supp} \psi_{0}$ and $|\hat{x} \cdot \hat{\xi}|>1-\varepsilon$ for some $0<\varepsilon<1$ and sufficiently large $|x|$. Letting

$$
p(x, \xi)=\chi(x, \xi) \psi_{0}\left(|\xi|^{2}\right) \psi(\xi /|\xi|)
$$

we consider a Ps.D.Op. $P$ with symbol $p(x, \xi): P=p\left(x, D_{x}\right)$, and define

$$
\begin{gathered}
G=H P-P H_{0}, \\
Q_{\alpha}=\left(H-H_{\alpha}\right) J_{\alpha}=\sum_{\gamma \neq \alpha} V_{\gamma} J_{\alpha} .
\end{gathered}
$$

We define the trace of the Fourier transform by $\left(\mathscr{F}_{0}(\lambda) f\right)(\theta)=\left(\mathscr{F}_{0} f\right)(\lambda, \theta)$ and $\left(\mathscr{F}_{\alpha}(\lambda) f\right)(\omega)=\left(\mathscr{F}_{\alpha} f\right)(\lambda, \omega)$ for $f \in L^{2, s}, s>1 / 2$. Let $\langle$,$\rangle be the inner product of L^{2}\left(S^{5}\right)$ and $\varphi(\xi)=\psi_{0}\left(|\xi|^{2}\right) \psi(\xi /|\xi|)$.

Lemma 3.1. Let $\hat{f} \in C_{0}^{\infty}\left(\left(E^{\alpha}, \infty\right) ; C^{\infty}\left(S^{2}\right)\right)$ and $\hat{g} \in C_{0}^{\infty}\left((0, \infty) ; C^{\infty}\left(S^{5}\right)\right)$. Then

$$
\begin{aligned}
\left(\mathscr{F}_{0} \varphi\left(D_{x}\right) S_{0 \alpha} \mathscr{F}_{\alpha}^{*} \hat{f}, \hat{g}\right)= & -2 \pi i \int_{0}^{\infty}\left\langle\mathscr{F}_{0}(\lambda) P^{*} Q_{\alpha} \mathscr{F}_{\alpha}^{*}(\lambda) \hat{f}(\lambda), \hat{g}(\lambda)\right\rangle d \lambda \\
& +\lim _{\varepsilon \downarrow 0} 2 \pi i \int_{0}^{\infty}\left\langle\mathscr{F}_{0}(\lambda) G^{*} R(\lambda+i \varepsilon) Q_{\alpha} \mathscr{F}_{\alpha}^{*}(\lambda) \hat{f}(\lambda), \hat{g}(\lambda)\right\rangle d \lambda .
\end{aligned}
$$

Proof. We put $f=\mathscr{F}_{\alpha}^{*} \hat{f}, g=\mathscr{F}_{0}^{*} \hat{g}$. By the stationary phase method and (3.1), we have

which implies

$$
\begin{aligned}
W_{0}^{+} \varphi\left(D_{x}\right) g & =\underset{t \rightarrow \infty}{s-\lim _{t \rightarrow \infty}} e^{i t H} \varphi\left(D_{x}\right) w^{-i t H_{0}} g \\
& =s-\lim _{t \rightarrow \infty} e^{i t H} P e^{-i t H_{0}} g
\end{aligned}
$$

$$
W_{0}^{+} \varphi\left(D_{x}\right) g=P g+i \int_{0}^{\infty} e^{i s H} G e^{-i s H_{0}} g d s
$$

On the other hand, letting $h_{\alpha}=-\Delta_{x_{\alpha}}+E^{\alpha}$, we have $e^{-i t H_{\alpha}} J_{\alpha}=J_{\alpha} e^{-i t h_{x}}$. Therefore,

$$
W_{\alpha}^{+}-W_{\alpha}^{-}=i \int_{-\infty}^{\infty} e^{i t H} Q_{\alpha} e^{-i t h_{x}} d t
$$

Since the ranges of $W_{0}^{+}$and $W_{\alpha}^{+}$are orthogonal, $\left(W_{0}^{+}\right)^{*} W_{\alpha}^{+}=0$, hence.

$$
S_{0_{\alpha}}=\left(W_{0}^{+}\right)^{*} W_{\alpha}^{-}=\left(W_{0}^{+}\right)^{*}\left(W_{\alpha}^{-}-W_{\alpha}^{+}\right) .
$$

This formula and (3.6) imply that

$$
\left(\varphi\left(D_{x}\right) S_{0 \alpha} f, g\right)=-i \int_{-\infty}^{\infty}\left(e^{i t H} Q_{\alpha} e^{-i t h_{x}} f, W_{0}^{+} \varphi\left(D_{x}\right) g\right) d t .
$$

In view of (3.5), (3.7) and the interwining property, $e^{-i t H} W_{0}^{+}=W_{0}^{+} e^{-i t H_{0}}$, we have

$$
\begin{aligned}
\left(\varphi\left(D_{x}\right) S_{0 \alpha} f, g\right)= & -i \int_{-\infty}^{\infty} d t\left(Q_{\alpha} e^{-i t h_{x}} f, P e^{-i t H_{0}} g\right) \\
& -\int_{0}^{\infty} d s \int_{-\infty}^{\infty} d t\left(Q_{\alpha} e^{-i t h_{x}} f, e^{i s H} G e^{-i(s+t) H_{0}} g\right)
\end{aligned}
$$


Passing to the Fourier transforms, we obtain

$$
\begin{aligned}
& \int_{-\infty}^{\infty} d t\left(G^{*} e^{-i s H} Q_{\alpha} e^{-i t h_{\alpha}} f, e^{-i(s+t) H_{0}} g\right) \\
& =\int_{-\infty}^{\infty} d t \int_{0}^{\infty} d \lambda\left\langle\mathscr{F}_{0}\left[G^{*} e^{-i s H} Q_{\alpha} e^{-i t h_{\alpha}} f\right](\lambda), e^{-i(s+t) \lambda} \hat{g}(\lambda)\right\rangle .
\end{aligned}
$$

To calculate the integral with respect to $t$, we introduce a convergent factor $e^{-\varepsilon|t|}$ and let $\varepsilon \rightarrow 0$. Then the above integral is equal to

$$
\begin{gathered}
2 \pi \int_{0}^{\infty} d \lambda\left\langle\mathscr{F}_{0}\left[G^{*} e^{-i s(H-\lambda)} Q_{\alpha} e_{\alpha}^{\prime}(\lambda) f\right](\lambda), \hat{g}(\lambda)\right\rangle, \\
e_{\alpha}^{\prime}(\lambda)=\frac{1}{2 \pi i}\left(\left(h_{\alpha}-\lambda-i 0\right)^{-1}-\left(h_{\alpha}-\lambda+i 0\right)^{-1}\right) .
\end{gathered}
$$

Thus the second term on the right-hand side of (3.8) is equal to

$$
-2 \pi \int_{0}^{\infty} d s \int_{0}^{\infty} d \lambda\left\langle\mathscr{F}_{0}\left[G^{*} e^{-i s(H-\lambda)} Q_{\alpha} e_{\alpha}^{\prime}(\lambda) f\right](\lambda), \hat{g}(\lambda)\right\rangle .
$$

We again insert the convergent factor $e^{-\varepsilon s}$ and let $\varepsilon \rightarrow 0$. Then the above integral equals

$$
2 \pi i \lim _{\varepsilon \downarrow 0} \int_{0}^{\infty}\left\langle\mathscr{F}_{0}(\lambda) G^{*} R(\lambda+i \varepsilon) Q_{\alpha} e_{\alpha}^{\prime}(\lambda) f, \hat{g}(\lambda)\right\rangle d \lambda .
$$

Since $e_{\alpha}^{\prime}(\lambda)=\mathscr{F}_{\alpha}^{*}(\lambda) \mathscr{F}_{\alpha}(\lambda),(3.9)$ is written as

$$
2 \pi i \lim _{\varepsilon \downarrow 0} \int_{0}^{\infty}\left\langle\mathscr{F}_{0}(\lambda) G^{*} R(\lambda+i \varepsilon) Q_{\alpha} \mathscr{F}_{\alpha}^{*}(\lambda) \hat{f}(\lambda), \hat{g}(\lambda)\right\rangle d \lambda .
$$

Arguing quite similarly, one can show that the first term on the right-hand side of (3.8) is written as

$$
-2 \pi i \int_{0}^{\infty}\left\langle\mathscr{F}_{0}(\lambda) P^{*} Q_{\alpha} \mathscr{F}_{\alpha}^{*}(\lambda) \hat{f}(\lambda), \hat{g}(\lambda)\right\rangle d \lambda
$$

If one can give a define meaning to the operator $G^{*} R(\lambda+i 0) Q_{\alpha}$ and exchange the order of integration in $\lambda$ and lim, one obtains

$$
\begin{aligned}
\psi(\theta) \hat{S}_{0 \alpha}(\lambda)= & -2 \pi i \mathscr{F}_{0}(\lambda) P^{*} Q_{\alpha} \mathscr{F}_{\alpha}^{*}(\lambda) \\
& +2 \pi i \mathscr{F}_{0}(\lambda) G^{*} R(\lambda+i 0) Q_{\alpha} \mathscr{F}_{\alpha}^{*}(\lambda)
\end{aligned}
$$

if $\lambda \in I$. The justification of this procedure is the subject of the next section.

\section{Proof of Theorem 1.1}

4.1. Decomposition of $\hat{S}_{0 \alpha}$. For sufficiently small $\varepsilon>0$, we set

$$
\begin{aligned}
X_{\beta}^{\varepsilon} & =\left\{x \in X ;\left|x^{\beta}\right| /|x|<\varepsilon\right\}, \\
M^{\varepsilon} & =S^{5} \backslash \cup_{\beta} X_{\beta}^{\varepsilon}, \\
N^{\varepsilon} & =S^{5} \cap\left(\cup_{\beta} X_{\beta}^{\varepsilon}\right) .
\end{aligned}
$$


We take $\psi_{M}(\theta), \psi_{N}(\theta) \in C^{\infty}\left(S^{5}\right)$ such that

$$
\begin{gathered}
\psi_{M}(\theta)+\psi_{N}(\theta)=1, \\
\psi_{M}(\theta)= \begin{cases}1 & \theta \in M^{2 \varepsilon}, \\
0 & \theta \in N^{\varepsilon},\end{cases} \\
\psi_{N}(\theta)= \begin{cases}1 & \theta \in N^{\varepsilon}, \\
0 & \theta \in M^{2 \varepsilon}\end{cases}
\end{gathered}
$$

We put

$$
\begin{aligned}
& \hat{S}_{M}(\lambda)=\psi_{M}(\theta) \hat{S}_{0 \alpha}(\lambda), \\
& \hat{S}_{N}(\lambda)=\psi_{N}(\theta) \hat{S}_{0 \alpha}(\lambda) .
\end{aligned}
$$

We also prepare localizations in $x$-space. We take $\chi_{M}(x), \chi_{N}(x) \in C^{\infty}(X)$ homogeneous of degree 0 for $|x|>1$ and

$$
\begin{aligned}
& \chi_{M}(x)=\left\{\begin{array}{ll}
1 & \text { if } \hat{x} \in M^{\varepsilon / 2}, \\
0 & \text { if } \hat{x} \in N^{\varepsilon / 4},
\end{array},\right. \\
& \chi_{N}(x)= \begin{cases}1 & \text { if } \hat{x} \in N^{2 \varepsilon}, \\
0 & \text { if } \hat{x} \in M^{3 \varepsilon},\end{cases}
\end{aligned}
$$

where $\hat{x}=x /|x|$. The important properties of these localizations are as follows.

Lemma 4.1. (1) $\operatorname{supp} \nabla \chi_{M}, \operatorname{supp} \nabla \chi_{N} \subset X \backslash \cup_{\beta} X_{\beta}$.

(2) $\operatorname{supp} \nabla \chi_{M} \cap \operatorname{supp} \psi_{M}=\varnothing$, supp $\nabla \chi_{N} \cap \operatorname{supp} \psi_{N}=\varnothing$, if $|x|>1$.

Note that in Lemma 4.1 (2), we extend $\psi_{M}, \psi_{N}$ on $X-\{0\}$ as homogeneous functions of degree 0 . One should also note that if $\varepsilon$ is sufficiently small, $\chi_{N}(x)$ is split into three parts:

$$
\begin{aligned}
& \chi_{N}(x)=\sum_{\beta} \chi_{B}(x), \\
& \chi_{\beta}(x)= \begin{cases}1 & \text { if } x \in X_{\beta}^{2 \varepsilon}, \\
0 & \text { if } x \notin X_{\beta}^{3 \varepsilon} .\end{cases}
\end{aligned}
$$

We next localize the energy. We fix $\lambda>0$ arbitrarily and for small $\varepsilon_{1}>0$ we take $\psi_{1}(t) \in C_{0}^{\infty}\left(\mathbf{R}^{1}\right)$ such that

$$
\psi_{1}(t)=\left\{\begin{array}{ll}
1 & \text { if }|t-\lambda|<\varepsilon_{1} \\
0 & \text { if }|t-\lambda|>2 \varepsilon_{1}
\end{array} .\right.
$$

4.2. Continuity of the Kernel of $\hat{S}_{M}(\lambda)$. With $\chi_{M}, \psi_{M}, \psi_{1}$ as above, we put

$$
p(x, \xi)=\chi_{M}(x) \psi_{M}(\xi /|\xi|) \psi_{1}\left(|\xi|^{2}\right) \text {. }
$$

Let $P$ be a Ps.D.Op. with symbol $p(x, \xi)$ and $G=H P-P H_{0}$. We look at the formula in Lemma 3.1. It is rather easy to see that if $\rho>3, \mathscr{F}_{0}(\lambda) P^{*} Q_{\alpha} \mathscr{F}_{\alpha}^{*}(\lambda)$ has a continuous kernel. In fact letting

$$
f=\sum_{\gamma \neq \alpha} V_{\gamma}\left(x^{\gamma}\right) u_{\alpha}\left(x^{\alpha}\right)^{i \sqrt{\lambda-E^{\alpha}} \omega \cdot x_{\alpha}}
$$


one can see that the above operator has an integral kernel

$$
C_{0}(\lambda) C_{\alpha}(\lambda) \int_{\mathbf{R}^{6}} e^{-i \sqrt{\lambda} \theta \cdot x}\left(P^{*} f\right)(x) d x .
$$

Since $u_{\alpha}$ decays exponentially, we have

$$
|f| \leqq C_{k}\left\langle x^{\alpha}\right\rangle^{-k}\left\langle x_{\alpha}\right\rangle^{-\rho} \text { for any } k>1,
$$

which shows that (4.5) is continuous with respect to $\lambda>0, \theta \in S^{5}$ and $\omega \in S^{2}$ if $\rho>3$.

For notational convenience, for a Ps.D.Op. $A$, we write $A=O\left(\langle x\rangle^{m}\right)$, if its symbol $a(x, \xi)$ satisfies $\left|\partial_{x}^{k} \partial_{\xi}^{n} a(x, \xi)\right| \leqq C_{k n}\langle x\rangle^{m-k}$ for all $k, n$. Now, since $G=\left[H_{0}, P\right]+$ $\Sigma_{\beta} V_{\beta} P$, by virtue of our localization $\chi_{M}(x)$ and Lemma 4.1 , we have

$$
G^{*}=\langle x\rangle^{-1} P_{-}+O\left(\langle x\rangle^{-\rho}\right)
$$

where $P_{-}$verifies the assumptions in Theorem 2.2. For small $\varepsilon>0$, consider

$$
\begin{aligned}
G^{*} R(\lambda+i 0) f= & \langle x\rangle^{-3-\varepsilon_{\cdot}}\langle x\rangle^{2+\varepsilon} P_{-} R(\lambda+i 0)\langle x\rangle^{-3-2 \varepsilon_{\cdot}}\langle x\rangle^{3+2 \varepsilon} f \\
& +\langle x\rangle^{-3-\varepsilon} \cdot O\left(\langle x\rangle^{-\rho+3+\varepsilon}\right) R(\lambda+i 0)\langle x\rangle^{-1 / 2-\varepsilon_{\cdot}}\langle x\rangle^{1 / 2+\varepsilon} f .
\end{aligned}
$$

Then by Theorem 2.2 and (4.6), if $\rho>4+1 / 2$, the right-hand side is in $L^{1}\left(\mathbf{R}^{6}\right)$, hence

$$
C_{0}(\lambda) C_{\alpha}(\lambda) \int_{\mathbf{R}^{6}} e^{-i \sqrt{\lambda} \theta \cdot x} G^{*} R(\lambda+i 0) f d x
$$

is continuous with respect to $\lambda>0, \theta \in S^{5}$ and $\omega \in S^{2}$, which in turn implies that $\mathscr{F}_{0}(\lambda) G^{*} R(\lambda+i 0) Q_{\alpha} \mathscr{F}_{\alpha}^{*}(\lambda)$ has a continuous kernel (4.7).

We note that, since $P^{*}-G^{*} R(\lambda+i 0)=\left(H_{0}-\lambda\right) P^{*} R(\lambda+i 0)$, the kernel of $\hat{S}_{M}(\lambda)$ is given by

$$
-2 \pi i C_{\alpha}(\lambda) \mathscr{F}_{0}(\lambda)\left(H_{0}-\lambda\right) P^{*} R(\lambda+i 0) f .
$$

\subsection{Singularities of the Kernel of $\hat{S}_{N}(\lambda)$.}

4-3-1. Localization. To calculate the kernel of $\hat{S}_{N}(\lambda)$, we again make use of Lemma 3.1. However, in this case, we must be careful in choosing $p(x, \xi)$. First we note that on the support of $\psi_{1}\left(|\xi|^{2}\right) \psi_{N}(\xi /|\xi|),\left|\xi^{\beta}\right| /|\xi|<2 \varepsilon,\left.|| \xi\right|^{2}-\lambda \mid<2 \varepsilon_{1}$. We take $\psi_{\beta}(t) \in C_{0}^{\infty}\left(\mathbf{R}^{1}\right)$ such that

$$
\psi_{\beta}(t)= \begin{cases}1 & |t-\lambda|<\varepsilon_{2}, \\ 0 & |t-\lambda|>2 \varepsilon_{2} .\end{cases}
$$

Then by an appropriate choice of $\varepsilon$ 's, we have

$$
\psi_{1}\left(|\xi|^{2}\right) \psi_{N}(\xi /|\xi|)=\sum_{\beta} \psi_{\beta}\left(\left|\xi_{\beta}\right|^{2}\right) \psi_{1}\left(|\xi|^{2}\right) \psi_{N}(\xi /|\xi|)
$$

With $\chi_{\beta}(x)$ introduced in (4.3), we define

$$
p(x, \xi)=\sum_{\beta} \chi_{\beta}(x) \psi_{\beta}\left(\left|\xi_{\beta}\right|^{2}\right) \psi_{1}\left(|\xi|^{2}\right) \psi_{N}(\xi /|\xi|) \text {. }
$$

For a suitable choice of $\varepsilon$ 's, $\operatorname{supp} \nabla \chi_{\beta}$ and $\operatorname{supp} \psi_{\beta}\left(\left|\xi_{\beta}\right|^{2}\right) \psi_{1}\left(|\xi|^{2}\right)$ are disjoint. Let $P$ be a Ps.D.Op. with symbol $p(x, \xi)$ defined by (4.10) and $G=H P-P H_{0}$. Then, since $G=\left[H_{0}, P\right]+\Sigma_{\beta} V_{\beta} P$, we have

$$
G^{*}=\langle x\rangle^{-1} P_{-}+O\left(\langle x\rangle^{-k}\right)+P^{*} \sum_{\beta} V_{\beta},
$$




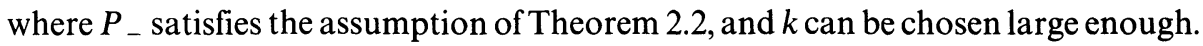
4-3-2. Continuous Parts. We apply Lemma 3.1. Then by the same reasoning as in 4-2, we see that the operators $\mathscr{F}_{0}(\lambda) P^{*} Q_{\alpha} \mathscr{F}_{\alpha}^{*}(\lambda)$ and $\mathscr{F}_{0}(\lambda)\langle x\rangle^{-1} P_{-} R(\lambda+i 0) Q_{\alpha} \mathscr{F}_{\alpha}^{*}(\lambda)$ have continuous kernels if $\rho>4+1 / 2$. symbol

We next study $\lim _{\kappa \downarrow 0} \mathscr{F}_{0}(\lambda) P^{*} \Sigma_{\beta} V_{\beta} R(\lambda+i \kappa) Q_{\alpha} \mathscr{F}_{\alpha}^{*}(\lambda)$. Let $A_{\beta}$ be the Ps.D.Op. with

$$
\chi_{\beta}(x) \psi_{\beta}\left(\left|\xi_{\beta}\right|^{2}\right) \psi_{1}\left(|\xi|^{2}\right) \psi_{N}(\xi /|\xi|) \text {. }
$$

Then $P=\Sigma_{\beta} A_{\beta}$, and if $\beta \neq \gamma, A_{\beta}^{*} V_{\gamma}=O\left(\langle x\rangle^{-\rho}\right)$. Therefore, as has been proved in $4-2$, if $\rho>4+1 / 2$ and $\beta \neq \gamma, \mathscr{F}_{0}(\lambda) A_{\beta}^{*} V_{\gamma} R(\lambda+i 0) Q_{\alpha} \mathscr{F}_{\alpha}^{*}(\lambda)$ has a continuous kernel.

4-3-3. Singular Parts. It remains to consider $\lim _{\kappa \downarrow 0} \mathscr{F}_{0}(\lambda) A_{\beta}^{*} V_{\beta} R(\lambda+i \kappa) Q_{\alpha} \mathscr{F}_{\alpha}^{*}(\lambda)$. In this case we must be careful in taking the limit, since $A_{\beta}^{*} V_{\beta}$ does not decay in $x_{\beta}$. Letting $\Psi_{N}(\xi)=\psi_{1}\left(|\xi|^{2}\right) \psi_{N}(\xi /|\xi|)$, we have

$$
A_{\beta}^{*}=\Psi_{N}\left(D_{x}\right) \psi_{\beta}\left(D_{x_{\beta}}\right) \chi_{\beta}(x)
$$

where $\psi_{\beta}\left(D_{x_{\beta}}\right)$ denotes the Ps.D.Op. with symbol $\psi_{\beta}\left(\left|\xi_{\beta}\right|^{2}\right)$. Noting that $\psi_{\beta}\left(D_{x_{\beta}}\right) \chi_{\beta}(x)$ commutes with $V_{\beta}$, we consider $\psi_{\beta}\left(D_{x_{\beta}}\right) \chi_{\beta}(x) R(\lambda+i \kappa) Q_{\alpha} \mathscr{F}_{\alpha}^{*}(\lambda)$.

Lemma 4.2. Let $f$ be defined by (4.4), $z=\lambda+i \kappa$ and $g=\left(H_{\beta}-z\right) \psi_{\beta}\left(D_{x_{\beta}}\right) \chi_{\beta}(x) R(z) f$. Then $g \in L^{2, s}$ for any $s<\rho-3 / 2$ uniformly in $\kappa \geqq 0$.

Proof. Letting $u=R(z) f$, we have

$$
\begin{aligned}
g & =\psi_{\beta}\left(D_{x_{\beta}}\right) \chi_{\beta}(x) f-\psi_{\beta}\left(D_{x_{\beta}}\right) \chi_{\beta}(x) \sum_{\gamma \neq \beta} V_{\gamma} u+\psi_{\beta}\left(D_{x_{\beta}}\right)\left[H_{0}, \chi_{\beta}\right] u \\
& =g_{1}+g_{2}+g_{3} .
\end{aligned}
$$

It easily follows from (4.6) that $g_{1} \in L^{2, s}, \forall s<\rho-3 / 2$ and $g_{2} \in L^{2, s}, \forall s<\rho-1 / 2$. We take $\varphi \in C_{0}^{\infty}\left(\mathbf{R}^{1}\right)$ such that for small $\varepsilon_{3}>0$,

Then as is well-known,

$$
\varphi(t)= \begin{cases}1 & |t-\lambda|<\varepsilon_{3} \\ 0 & |t-\lambda|>2 \varepsilon_{3} .\end{cases}
$$

$$
(1-\varphi(H)) R(\lambda+i \kappa) \in \mathbf{B}\left(L^{2, s} ; L^{2, s}\right)
$$

for any $s>1 / 2$ uniformly in $\kappa \geqq 0$. Therefore

$$
\psi_{\beta}\left(D_{x_{\beta}}\right)\left[H_{0}, \chi_{\beta}\right](1-\varphi(H)) R(\lambda+i \kappa) f \in L^{2, s}
$$

for any $s<\rho-1 / 2$ uniformly in $\kappa \geqq 0$. Lemma 2.1 implies that

$$
\left[H_{0}, \chi_{\beta}\right] \varphi(H) R(\lambda+i \kappa)=\left[H_{0}, \chi_{\beta}\right] \varphi\left(H_{0}\right) R(\lambda+i \kappa)+O\left(\langle x\rangle^{-\rho-1}\right) R(\lambda+i \kappa) .
$$

Now, on the support of the symbol of $\psi_{\beta}\left(D_{x_{\beta}}\right)\left[H_{0}, \chi_{\beta}\right] \varphi\left(H_{0}\right),\left.|| \xi_{\beta}\right|^{2}-\lambda \mid \leqq 2 \varepsilon_{2}$, $\left.|| \xi\right|^{2}-\lambda \mid \leqq 2 \varepsilon_{1}$ and $\left|x_{\beta}\right| /|x| \geqq 2 \varepsilon$. If $\varepsilon_{1}$ and $\varepsilon_{2}$ are chosen small enough, $\xi$ is localized near the $X_{\beta}$-plane, which shows that $\langle x\rangle \psi_{\beta}\left(D_{x_{\beta}}\right)\left[H_{0}, \chi_{\beta}\right] \varphi\left(H_{0}\right)$ satisfies the assumptions of Theorem 2.2. Then we have $g_{3} \in L^{2, s}$ for any $s<\rho-3 / 2$ uniformly in $\kappa \geqq 0$.

Using Lemma 4.2, letting $R_{\beta}(z)=\left(H_{\beta}-z\right)^{-1}$, we have

$$
\psi_{\beta}\left(D_{x_{\beta}}\right) \chi_{\beta}(x) R(\lambda+i \kappa) f=R_{\beta}(\lambda+i \kappa) g,
$$


whence

$$
\mathscr{F}_{0}(\lambda) A_{\beta}^{*} V_{\beta} R(\lambda+i \kappa) f=\mathscr{F}_{0}(\lambda) \Psi_{N}\left(D_{x}\right) V_{\beta} R_{\beta}(\lambda+i \kappa) g .
$$

Note that $\Psi_{N}(\sqrt{\lambda} \theta)=1$ if $\left|\theta^{\beta}\right|$ is sufficiently small. Recall that $\mathscr{F}_{0}(\lambda)$ is the Fourier transformation followed by the restriction to the sphere of radius $\sqrt{\lambda}$. Thus integrating $V_{\beta} R_{\beta}(\lambda+i \kappa) g$ with respect to $x_{\beta}$ first and to $x^{\beta}$ later and letting $r_{\beta}(z)=$ $\left(-\Delta_{x^{\beta}}+V_{\beta}-z\right)^{-1}$, we see that (4.12) is equal to

$$
\begin{gathered}
C_{0}(\lambda) \int_{\mathbf{R}^{3}} e^{-i \sqrt{\lambda} \theta^{\beta} \cdot x^{\beta}} V_{\beta} r_{\beta}\left(\lambda\left|\theta^{\beta}\right|^{2}+i \kappa\right) \hat{g} d x^{\beta}, \\
\hat{g}=\hat{g}\left(x^{\beta} ; \lambda, \theta_{\beta}, \omega\right)=\int_{\mathbf{R}^{3}} e^{-i \sqrt{\lambda} \theta_{\beta} \cdot x_{\beta}} g\left(x^{\beta}, x_{\beta}\right) d x_{\beta} .
\end{gathered}
$$

If $\rho>5+1 / 2, \hat{g}\left(\cdot ; \lambda, \theta_{\beta}, \omega\right)$ is an $L^{2, s}\left(\mathbf{R}^{3}\right)$-valued continuous function of $\lambda, \theta_{\beta}$ and $\omega$ for some $s>5 / 2$. In fact, noting that for small $\varepsilon>0$,

$$
\left|\hat{g}\left(x^{\beta}\right)\right|^{2} \leqq C \int_{\mathbf{R}^{3}}\left\langle x_{\beta}\right\rangle^{3+\varepsilon}\left|g\left(x^{\beta}, x_{\beta}\right)\right|^{2} d x_{\beta},
$$

we have

$$
\int_{\mathbf{R}^{3}}\left\langle x^{\beta}\right\rangle^{2 s}\left|\hat{g}\left(x^{\beta}\right)\right|^{2} d x^{\beta} \leqq C \int_{\mathbf{R}^{6}}\langle x\rangle^{2 s+3+\varepsilon}\left|g\left(x^{\beta}, x_{\beta}\right)\right|^{2} d x .
$$

Since $g \in L^{2, s}$ for any $s<\rho-3 / 2$, the last integral converges for some $s>5 / 2$ if $\rho>5+1 / 2$.

We are now in a position to find the singularities of the kernel of $\hat{S}_{N}(\lambda)$. By using (2.10), we have

$$
\begin{aligned}
\int_{\mathbf{R}^{3}} e^{-i \sqrt{\lambda} \theta^{\beta} \cdot x^{\beta}} V_{\beta} r_{\beta}\left(\lambda\left|\theta^{\beta}\right|^{2}+i \kappa\right) \hat{g} d x^{\beta} \simeq & \left(\lambda\left|\theta^{\beta}\right|^{2}\right)^{-1} \int_{\mathbf{R}^{3}} e^{-i \sqrt{\lambda} \theta^{\beta} \cdot x^{\beta}} V_{\beta} B_{-2} \hat{g} d x^{\beta} \\
& +\left(\lambda\left|\theta^{\beta}\right|^{2}\right)^{-1 / 2} \int_{\mathbf{R}^{3}} e^{-i \sqrt{\lambda} \theta^{\beta} \cdot x^{\beta}} V_{\beta} B_{-1} \hat{g} d x^{\beta}
\end{aligned}
$$

as $\kappa \rightarrow 0$. Let $u_{\beta}^{(j)}$ be normalized eigenfunctions of $h^{\beta}$ with zero eigenvalue. Then $B_{-2} \hat{g}=\Sigma_{j} A_{\beta 1}^{(j)}\left(\lambda ; \theta_{\beta}, \omega\right) u_{\beta}^{(j)}\left(x^{\beta}\right)$ with

$$
A_{\beta}^{(j)}\left(\lambda ; \theta_{\beta}, \omega\right)=-\int_{\mathbf{R}^{3}} \hat{g}\left(x^{\beta} ; \lambda, \theta_{\beta}, \omega\right) u_{\beta}^{(j)}\left(x^{\beta}\right) d x^{\beta} .
$$

In view of Lemma $2.3(1)$, as $\left|\theta^{\beta}\right| \rightarrow 0$, we see that the first term of the right-hand side of (4.15) behaves like

$$
-i\left(\sqrt{\lambda}\left|\theta^{\beta}\right|\right)^{-1} \sum_{j} A_{\beta 1}^{(j)}\left(\lambda ; \theta_{\beta}, \omega\right) \int_{\mathbf{R} 3} \frac{\theta^{\beta}}{\left|\theta^{\beta}\right|} \cdot x_{\beta} V_{\beta}\left(x^{\beta}\right) u_{\beta}^{(j)}\left(x^{\beta}\right) d x^{\beta}+O(1) .
$$

We have thus found the contribution of the zero-eigenvalue of $h^{\beta}$ to the singularities. By a similar calculation one can also find the contribution of the zero-resonances. In particular, we have shown that $C_{\beta 1}^{(j)}\left(\lambda ; \theta_{\beta}, \omega\right)$ and $C_{\beta 2}\left(\lambda ; \theta_{\beta}, \omega\right)$ are given by

$$
\begin{aligned}
& C_{\beta 1}^{(j)}\left(\lambda ; \theta_{\beta}, \omega\right)=2 \pi \lambda^{-1 / 2} C_{0}(\lambda) C_{\alpha}(\lambda) \int_{\mathbf{R}^{3}} \hat{g}\left(x^{\beta} ; \lambda, \theta_{\beta}, \omega\right) u_{\beta}^{(j)}\left(x^{\beta}\right) d x^{\beta}, \\
& C_{\beta 2}\left(\lambda ; \theta_{\beta}, \omega\right)=2 \pi \lambda^{-1 / 2} C_{0}(\lambda) C_{\alpha}(\lambda) \int_{\mathbf{R}^{3}} \hat{g}\left(x^{\beta} ; \lambda, \theta_{\beta}, \omega\right) \varphi_{\beta}\left(x^{\beta}\right) d x^{\beta} .
\end{aligned}
$$


We end this section by noting that as in (4.8) the kernel of $\hat{S}_{N}(\lambda)$ is given by

$$
-2 \pi i C_{\alpha}(\lambda) \mathscr{F}_{0}\left[\left(H_{0}-\lambda\right) P^{*} R(\lambda+i 0) f\right](\lambda),
$$

$P$ being defined by (4.10).

By examining the $\lambda$-dependence of all terms appearing above, one can show that $\hat{S}_{0 \alpha}(\lambda)$ is a $\mathbf{B}\left(L^{2}\left(S^{2}\right) ; L^{2}\left(S^{5}\right)\right)$-valued continuous function of $\lambda>0$.

\section{Proof of Theorem 1.2}

5.1. Scattering Amplitudes for 2-Cluster Scattering. We first recall the well-known formula for the scattering amplitudes for 2-cluster scattering. It is better to change the notation slightly. We denote by $a$ the triple $a=\left\{\alpha, E^{\alpha}, u_{\alpha}\right\}$, where $u_{\alpha}$ is a normalized eigenfunction of $h^{\alpha}$ with eigenvalue $E^{\alpha}$. We define the wave operators $W_{a}^{ \pm}$by (1.3) with $\alpha$ replaced by $a$ keeping $H_{\alpha}$ unchanged. For two triples $a=\left\{\alpha, E^{\alpha}, u_{\alpha}\right\}, b=$ $\left\{\beta, E^{\beta}, u_{\beta}\right\}$, we define the scattering operator $S_{b a}$ by $S_{b a}=\left(W_{b}^{+}\right)^{*} W_{a}^{-}$. We introduce the Fourier transformation $\mathscr{F}_{a}$ in the same way as in (1.7) and set $\hat{S}_{b a}=\mathscr{F}_{b} S_{b a} \mathscr{F}_{a}^{*}$. Then for any $\lambda>\max \left\{E^{\alpha}, E^{\beta}\right\}$, the S-matrix $\hat{S}_{b a}(\lambda) \in \mathbf{B}\left(L^{2}\left(S^{2}\right) ; L^{2}\left(S^{2}\right)\right)$ is defined similarly to $1-2$. The scattering amplitude $A_{b a}(\lambda)$ is defined by

$$
A_{b a}(\lambda)=\hat{S}_{b a}(\lambda)-\delta_{b a} \text {. }
$$

Let $Q_{a}=\Sigma_{\gamma \neq \alpha} V_{\gamma} J_{\alpha}$. Then we have, formally,

$$
A_{b a}(\lambda)=-2 \pi i \mathscr{F}_{b}(\lambda) J_{b}^{*} Q_{\alpha^{2}} \mathscr{F}_{a}^{*}(\lambda)+2 \pi i \mathscr{F}_{b}(\lambda) Q_{b}^{*} R(\lambda+i 0) Q_{a} \mathscr{F}_{a}^{*}(\lambda) .
$$

One can see that, if $\lambda \notin \Lambda, \rho>3, E^{\alpha}<0, E^{\beta}<0$, the right-hand side of (5.2) has a continuous kernel, up to a constant, given by

$$
\int_{\mathbf{R}^{6}} e^{-i \sqrt{\lambda-E^{\beta} \theta_{\beta} \cdot x_{\beta}}} u_{\beta}\left(x^{\beta}\right) f(x) d x-\int_{\mathbf{R}^{6}} e^{-i \sqrt{\lambda-E^{\beta}} \theta_{\beta} \cdot x_{\beta}} u_{\beta}\left(x^{\beta}\right) \sum_{\gamma \neq \beta} V_{\gamma} R(\lambda+i 0) f d x,
$$

$f$ being defined by (4.4). However if $E^{\beta}=0$, it is not obvious to give a definite meaning to the right-hand side of (5.2), since $u_{\beta}\left(x^{\beta}\right)$ does not decay sufficiently rapidly. In the next section, we derive a representation formula for the 2-cluster scattering amplitude when $E^{\beta}=0, E^{\alpha}<0$.

5.2. 2-Cluster Scattering Amplitudes for Zero Eigenvalue. Let $u_{\beta}$ be a normalized eigenfunction of $h^{\beta}$ with zero eigenvalue. Let $\chi_{\beta}(x)$ be defined by (4.3). In the following arguments, we always assume that $\rho>5+1 / 2$, and let $b=\left\{\beta, 0, u_{\beta}\right\}$.

Lemma 5.1. $\left(1-\chi_{\beta}(x)\right) e^{-i t H_{\beta}} J_{b} \rightarrow 0$ strongly as $|t| \rightarrow \infty$.

Proof. Since on the support of $1-\chi_{\beta},\left|x^{\beta}\right| \geqq \varepsilon|x|$ and $\left\langle x^{\beta}\right\rangle^{-1} \leqq C\left\langle x_{\beta}\right\rangle^{-1}$, we have by virtue of Lemma $2.3(2)$,

$$
\left|\left(1-\chi_{\beta}(x)\right) u_{\beta}\left(x^{\beta}\right)\right| \leqq C\left\langle x^{\beta}\right\rangle^{-3 / 2-\varepsilon}\left\langle x_{\beta}\right\rangle^{-1 / 2+\varepsilon} .
$$

The lemma then follows from the following inequality:

$$
\begin{aligned}
\left\|\left(1-\chi_{\beta}\right) e^{-i t H_{\beta}} J_{b} f\right\| & =\left\|\left(1-\chi_{\beta}\right) u_{\beta}\left(x^{\beta}\right)\left(e^{-i t h_{\beta}} f\right)\left(x_{\beta}\right)\right\| \\
& \leqq C\left\|\left\langle x^{\beta}\right\rangle^{-3 / 2-\varepsilon}\left\langle x_{\beta}\right\rangle^{-1 / 2+\varepsilon}\left(e^{-i t h_{\beta}} f\right)\left(x_{\beta}\right)\right\| .
\end{aligned}
$$


Let $\psi_{\beta}(t)$ be as in (4.9) and $\psi_{\beta}\left(D_{x_{\beta}}\right)$ be the Ps.D.Op with symbol $\psi_{\beta}\left(\left|\xi_{\beta}\right|^{2}\right)$. Let $P$ and $G$ be defined by

$$
P=\chi_{\beta}(x) \psi_{\beta}\left(D_{x_{\beta}}\right), \quad G=H P-P H_{\beta} .
$$

Using Lemma 5.1, one gets

$$
W_{b}^{+} \psi_{\beta}\left(D_{x_{\beta}}\right)=s-\lim _{t \rightarrow \infty} e^{i t H} P e^{-i t H_{\beta}} J_{b}=P J_{b}+i \int_{0}^{\infty} e^{i t H} G J_{b} e^{-i t h_{\beta}} d t
$$

Using (5.5), one can argue in the same way as in the proof of Lemma 3.1 to obtain

Lemma 5.2. Let $\hat{f}, \hat{g} \in C_{0}^{\infty}\left((0, \infty) ; C^{\infty}\left(S^{2}\right)\right)$, and $\left\langle\right.$, denote the inner product of $L^{2}\left(S^{2}\right)$.

Then we have

$$
\begin{aligned}
\left(\mathscr{F}_{b} \psi_{b}\left(D_{x_{\beta}}\right) S_{b a} \mathscr{F}{ }_{a}^{*} \hat{f}, \hat{g}\right)= & -2 \pi i \int_{0}^{\infty}\left\langle\mathscr{F}_{b}(\lambda) J_{b}^{*} P^{*} Q_{\alpha} \mathscr{F}_{a}^{*}(\hat{\lambda}) \hat{f}(\lambda), \hat{g}(\lambda)\right\rangle d \lambda \\
& +2 \pi i \int_{0}^{\infty}\left\langle\mathscr{F}_{b}(\lambda) J_{b}^{*} G^{*} R(\lambda+i 0) Q_{\alpha} \mathscr{F}_{a}^{*}(\lambda) \hat{f}(\lambda), \hat{g}(\lambda)\right\rangle d \lambda .
\end{aligned}
$$

Let $f$ be defined by (4.4). Then by the above lemma, $\hat{S}_{b a}(\lambda)$ is seen to have a kernel, at least formally,

$$
\hat{S}_{b a}\left(\lambda ; \theta_{\beta}, \omega\right)=-2 \pi i C_{\alpha}(\lambda) \mathscr{F}_{b}(\lambda) J_{b}^{*}\left(P^{*} f-G^{*} R(\lambda+i 0) f\right) .
$$

Let $v=R(\lambda+i 0) f$. Then

$$
P^{*} f-G^{*} R(\lambda+i 0) f=\left(H_{\beta}-\lambda\right) P^{*} v .
$$

By Lemma 4.2, the right-hand side is nothing but $g$ in the lemma with $z=\lambda+i 0$. Therefore,

$$
\hat{S}_{b a}\left(\lambda ; \theta_{\beta}, \omega\right)=-2 \pi i C_{\alpha}(\lambda) \mathscr{F}_{b}(\lambda) J_{b}^{*} g .
$$

Since $J_{b}^{*} g=\int_{\mathbf{R}^{3}} g(x) u_{\beta}\left(x^{\beta}\right) d x^{\beta}$, the right-hand side is equal to

$$
-2 \pi i C_{\alpha}(\lambda) C_{b}(\lambda) \int_{\mathbf{R}^{6}} e^{-i \sqrt{\lambda} \theta_{\beta} \cdot x_{\beta}} u_{\beta}\left(x^{\beta}\right) g(x) d x, \quad C_{b}(\lambda)=(2 \pi)^{-3 / 2} 2^{-1 / 2} \hat{\lambda}^{1 / 4} .
$$

Replacing $u_{\beta}$ by $u_{\beta}^{(j)}$ and using (4.17), we have,

$$
\hat{S}_{b a}\left(\lambda ; \theta_{\beta}, \omega\right)=-i(2 \pi)^{3 / 2} \lambda^{-1 / 4} C_{\beta 1}^{(j)}\left(\lambda ; \theta_{\beta}, \omega\right), \quad b=\left\{\beta, 0, u_{B}^{(j)}\right\} .
$$

5.3. Generalized Eigenfunction. We put

$$
\begin{aligned}
\varphi(x, \lambda, \omega) & =e^{i \sqrt{\lambda-E^{\alpha} \omega \cdot x_{x}}} u_{\alpha}\left(x^{\alpha}\right)-v, \\
v & =R(\lambda+i 0) f,
\end{aligned}
$$

$f$ being defined by (4.4). Then $H \varphi=\lambda \varphi$, namely, $\varphi(x, \lambda, \omega)$ is a generalized eigenfunction of the three-body Schrödinger operator $H$. The first term of the right-hand side of (5.11) represents the incident wave, and the second term the scattered wave. In the two-body problem, it is well-known that the scattering amplitude is obtained through the asymptotic behavior at infinity of $v$. This turns out to be the case for the three-body problem, which we shall study in our forthcoming paper. In this and the following subsections, we consider relations between $v$ and $C_{\beta 1}^{(j)}, C_{\beta 2}$. 
Let $P$ and $J_{b}$ be as in 5-2, and put

$$
w=J_{b}^{*} P^{*} v=\int_{\mathbf{R}^{3}} u_{\beta}\left(x^{\beta}\right) \psi_{\beta}\left(D_{x_{\beta}}\right) \chi_{\beta}(x) v(x) d x^{\beta} .
$$

Note that this makes sense since the integral is actually performed on the set $\left\{\left|x^{\beta}\right| \leqq 2 \varepsilon\left(1-4 \varepsilon^{2}\right)^{-1}\left|x_{\beta}\right|\right\}$. Then by a simple manipulation we have

$$
\left(-\Delta_{x_{\beta}}-\lambda\right) w=J_{b}^{*}\left(H_{\beta}-\lambda\right) P^{*} v=J_{b}^{*} g,
$$

where $g$ is as Lemma 4.2. Since $g \in L^{2, s} \forall s<\rho-3 / 2$, we see $J_{b}^{*} g \in L^{2, s} \forall s<\rho-3 / 2$. Therefore

$$
w=\left(-\Delta_{x_{\beta}}-\lambda-i 0\right)^{-1} J_{b}^{*} g .
$$

Here we recall the following fact for a relation between the Fourier transformation and the resolvent of the Laplacian.

Lemma 5.3. Let $R_{0}(z)$ be the resolvent of $-\Delta$ in $\mathbf{R}^{n}$ and $f \in L^{2,3 / 2}$. Let $C(\lambda)=$ $e^{(n-3) \pi i / 4} \pi^{-1 / 2} \lambda^{1 / 4}$. Then the following strong limit exists in $L^{2}\left(S^{n-1}\right)$ for any $\lambda>0$ :

$$
\mathscr{F}_{0}(\lambda) f=\mathrm{s}_{r \rightarrow \infty} \lim C(\lambda) r^{(n-1) / 2} e^{-i \sqrt{\lambda} r}\left(R_{0}(\lambda+i 0) f\right)(r \cdot)
$$

For the proof, see e.g. Saito [19] or [8]. Using this lemma, one finds the Fourier transform of $J_{b}^{*} g$ through the spatially asymptotic expansion of $v$, which is equal to $\hat{S}_{b a}\left(\lambda ; \theta_{\beta}, \omega\right)$ by $(5.8)$. We have thus proven

\section{Lemma 5.4 .}

$$
\begin{gathered}
\left(J_{b}^{*} P^{*} v\right)\left(r \theta_{\beta}\right) \sim C(\lambda) r^{-1} e^{i \sqrt{\lambda} r} C_{\beta 1}^{(j)}\left(\lambda ; \theta_{\beta}, \omega\right), \\
C(\lambda)=(2 \pi)^{5 / 2} \lambda^{-1 / 2}\left(\lambda-E^{\alpha}\right)^{-1 / 4}
\end{gathered}
$$

as $r=\left|x_{\beta}\right| \rightarrow \infty$ in $L^{2}\left(S^{2}\right)$, and

$$
C_{\beta 1}^{(j)}\left(\lambda ; \theta_{\beta}, \omega\right)=i(2 \pi)^{-3 / 2} \lambda^{1 / 4} \hat{S}_{b a}\left(\lambda ; \theta_{\beta}, \omega\right)
$$

5.4. Resonance Scattering. We use the terminology "resonance scattering" in a sense slightly different from that of physical literature (see e.g. [14]). One can think of the collision process, in which, after the collision, the pair $\beta$ takes the zero-resonance state. It is not easy to define the associated scattering amplitude by the time-dependent method, since the zero-resonance, $\varphi_{\beta}$, does not belong to $L^{2}\left(\mathbf{R}^{3}\right)$. However, the stationary method explained in 5-3 works equally well for this case. We define the operator $\tilde{J}_{b}^{*}$ by $\tilde{J}_{b}^{*} u=\int_{\mathbf{R}^{3}} \varphi_{\beta}\left(x^{\beta}\right) u(x) d x^{\beta}$ and set

$$
\bar{w}=\tilde{J}_{b}^{*} P^{*} v
$$

By the same reasoning as in (5.12), this makes sense. We then have

$$
\left(-\Delta_{x_{\beta}}-\lambda\right) \tilde{w}=\tilde{J}_{b}^{*} g .
$$

In view of the estimate $\left|\varphi_{\beta}\left(x^{\beta}\right)\right| \leqq C\left\langle x^{\beta}\right\rangle^{-1}$, we have $\tilde{J}_{b}^{*} g \in L^{2, s}\left(\mathbf{R}^{3}\right) \forall s<3$. One can then argue in the same way as above, using (4.18), to obtain 


\section{Lemma 5.5.}

$$
\begin{gathered}
\left(\tilde{J}_{b}^{*} P^{*} v\right)\left(r \theta_{\beta}\right) \sim C(\lambda) r^{-1} e^{i \sqrt{\lambda} r} C_{\beta 2}\left(\lambda ; \theta_{\beta}, \omega\right) \\
C(\lambda)=(2 \pi)^{5 / 2} \lambda^{-1 / 2}\left(\lambda-E^{\alpha}\right)^{-1 / 4}
\end{gathered}
$$

as $r=\left|x_{\beta}\right| \rightarrow \infty$ in $L^{2}\left(S^{2}\right)$.

Comparing Lemmas 5.4 and 5.5 , it seems to be natural to call $C_{\beta 2}\left(\lambda ; \theta_{\beta}, \omega\right)$ the scattering amplitude for the resonance scattering, up to a constant factor. We have thus completed the proof of Theorem 1.2.

5.5. Total Cross Section. The total cross section $\sigma_{a}(\lambda)$ is defined by

$$
\sigma_{a}(\lambda)=\left\|\hat{S}_{0 \alpha}(\lambda)\right\|_{\mathbf{H S}}^{2}+\sum_{b}\left\|\hat{S}_{b a}(\lambda)\right\|_{\mathbf{H S}}^{2}
$$

where $\|\cdot\|_{\mathrm{HS}}$ denotes the Hilbert-Schmidt norm and the summation ranges over all 2-body channels with initial state $a=\left\{\alpha, E^{\alpha}, u_{\alpha}\right\}$. As has been mentioned in the introduction, if $\rho>2, \sigma_{a}(\lambda)$ is known to be finite for a.e. $\lambda>0$, but whether $\sigma_{a}(\lambda)$ is finite or not for all $\lambda>0$ was still an open problem. The difficulties arise from the collision process from 2 to 3 clusters and also from the 2 cluster scattering amplitudes in which in the final state the pair $\beta$ takes the zero energy state. As a by-product of our results, however, we can overcome these difficulties under the assumption that $\rho>5+1 / 2$.

Theorem 5.6. If $\rho>5+1 / 2, \sigma_{a}(\lambda)<\infty$ for all $\lambda>0$.

Acknowledgements. Most of this work was completed during the author's stay at Paris-Nord University and Aårhus University. He is grateful to Professors J. C. Guillot and E. Balslev for their hospitality in Paris and Aårhus and also for helpful discussions.

\section{References}

1. Amrein, W. O., Pearson, D. B., Sinha, K. B.: Bounds on the total scattering cross-section for $N$-body systems. Nuovo Cim. 52 A, 115-131 (1979)

2. Amrein, W. O., Sinha, K. B.: On the three body scattering cross sections. J. Phys. A: Math. Gen. 15, 1567-1586 (1982)

3. Dolph, C. L., Macleod, J. B., Thoe, D.: The analytic continuation of the resolvent kernel and scattering operator associated with the Schrödinger operator. J. Math. Anal. Appl. 16, 311-332 (1966)

4. Enss, V.: Quantum scattering theory of two and three body systems with potentials of short and long range. Lecture Notes in Math. vol. 1159, Berlin, Heidelberg, New York: Springer 1985

5. Enss, V., Simon, B.: Finite total cross sections in non-relativistic quantum mechanics. Commun. Math. Phys. 76, 177-209 (1980)

6. Froese, R. G., Herbst, I.: Exponential bounds and absence of positive eigenvalues of $N$-body Schrödinger operators. Commun. Math. Phys. 87, 429-447 (1982)

7. Graf, G. M.: Asymptotic completeness for $N$-body short-range quantum systems: A new proof. Commun. Math. Phys. 132, 73-101 (1990)

8. Isozaki, H.: Eikonal equations and spectral representations for long-range Schrödinger Hamiltonians. J. Math. Kyoto Univ. 20, 243-261 (1980)

9. Isozaki, H.: Decay rates of scattering states for Schrödinger operators. J. Math. Kyoto Univ. 26, 595-603 (1986) 
10. Isozaki, H.: Asymptotic properties of generalized eigenfunctions for three body Schrödinger operators, preprint (1991)

11. Isozaki, H., Kitada, H.: Scattering matrices for two-body Schrödinger operators, Scientific Papers of the College of Arts and Sciences. Tokyo Univ. 35, 81-107 (1985)

12. Ito, H. T., Tamura, H.: Semi-classical asymptotics for total scattering cross sections of 3-body systems, preprint (1990)

13. Jensen, A., Kato, T.: Spectral properties of Schrödinger operators and time decay of the wave functions. Duke Math. J. 46, 583-611 (1979)

14. Landau, L. D., Lifshitz, E. M.: Quantum Mechanics, Non-relativistic Theory. Oxford, London, Edinburgh, New York, Paris, Frankfurt: Pergamon Press 1965

15. Mourre, E.: Absence of singular continuous spectrum of certain self-adjoint operators. Commun. Math. Phys. 78, 391-408 (1981)

16. Murata, M.: Asymptotic expansions in time for solutions of Schrödinger type equations. J. Funct. Anal. 49, 10-56 (1982)

17. Perry, P., Sigal, I. M., Simon, B.: Spectral analysis of $N$-body Schrödinger operators. Ann. Math. 114, 519-567 (1981)

18. Reed, M., Simon, B.: Methods of Modern Mathematical Physics, IV: Analysis of Operators. New York, San Francisco, London: Academic Press 1978

19. Saito, Y.: Spectral representation for the Schrödinger operator with long-range potentials. Lecture Notes in Math. vol. 727, Berlin, Heidelberg, New York: Springer 1979

20. Sigal, I. M., Soffer, A.: The $N$-particle scattering problem: Asymptotic completeness for short range quantum systems. Ann. Math. 125, 35-108 (1987)

21. Skibsted, E.: Propagation estimates of $N$-body Schrödinger operators. Commun. Math. Phys. 142, 67-98 (1991)

22. Yafaev, D.: On the multichannel scattering in two spaces. Theor. Math. Phys. 37, 867-874 (1978)

Communicated by H. Araki 